\title{
Combining Surface Chemistry with a FRET-Based Biosensor to Study the Dynamics of RhoA GTPase Activation in Cells on Patterned Substrates
}

Louis Hodgson, ${ }^{\dagger \neq}$ Eugene W.L. Chan, ${ }^{\dagger \S}$ Klaus M. Hahn** and Muhammad N. Yousaf ${ }^{\star \S}$

${ }^{\S}$ Department of Chemistry, ${ }^{\S}$ Carolina Center for Genome Science, ${ }^{\ddagger}$ Department of Pharmacology, University of North Carolina at Chapel Hill, Chapel Hill, North Carolina 27599-3290

\section{Supplementary Information.}

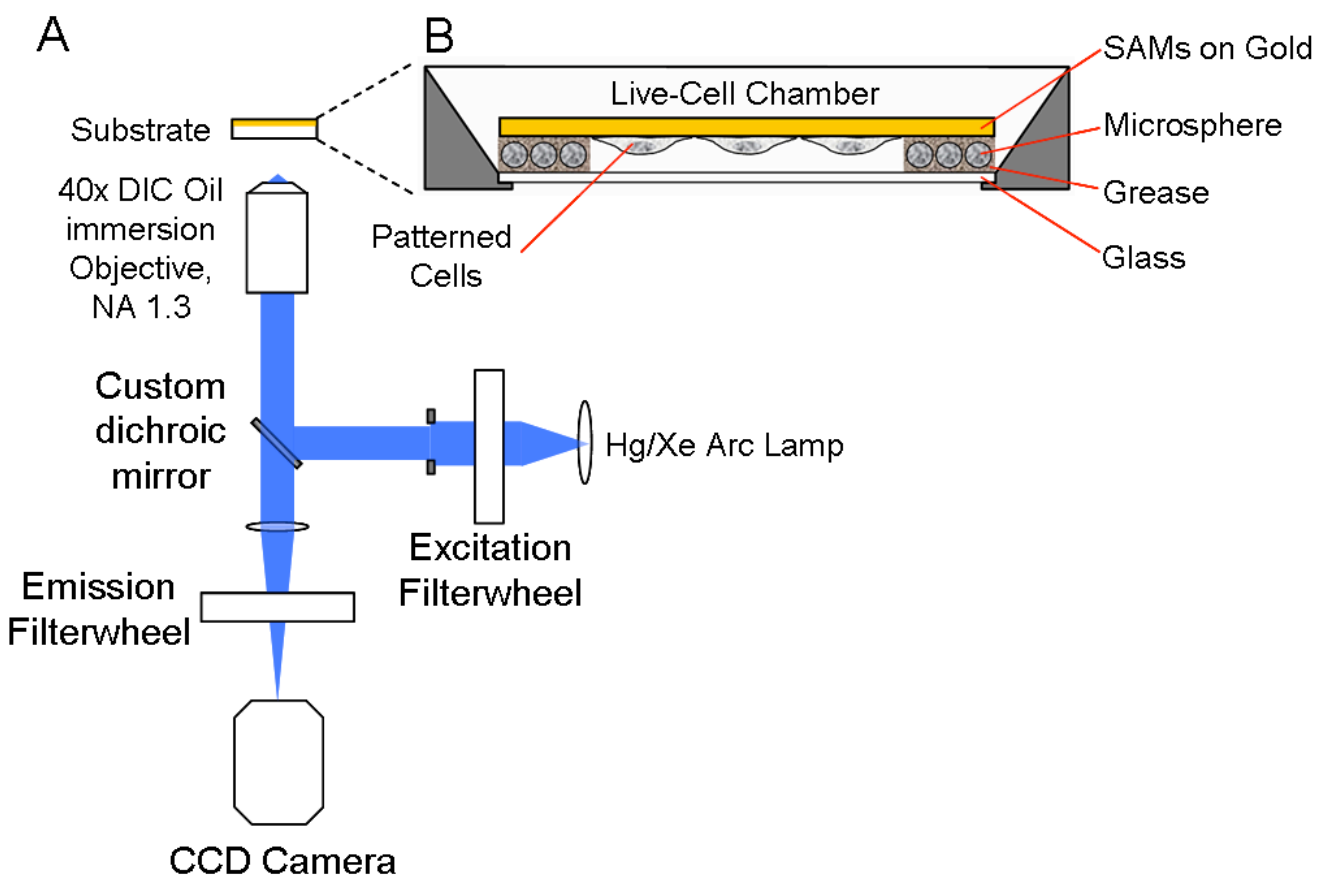

A. Schematic drawing of the wide-field epifluorescence microscope used for FRET experiments. An excitation light source ( $\mathrm{Hg} / \mathrm{Xe}$ arc lamp) illuminates the specimen through a 436/20nm bandpass filter and a custom-designed dichroic mirror. Emitted fluorescence is recorded on a cooled CCD camera (Roper/Photometrics CoolsnapESII) through emission band-pass filters (470/30nm for CFP and 535/30nm for FRET). A 40x oil-immersion, plan corrected NA 1.3 objective was used. B. Schematic drawing of the live-cell chamber customized to accommodate the gold-coated coverslips. Patterned substrate (yellow) is inverted and secured to a clear glass \#1.5 coverslip using a mixture of silicone vacuum grease mixed with 15 micron polystyrene microspheres. Defined diameter microspheres act as gaskets in keeping the patterned substrate off the surface of the clear coverslip. The sandwiched assembly is immersed in imaging medium and placed on a heated stage for experiments. 